\title{
Objective quantification of the impact of blepharoplasty on the superior visual field
}

\author{
Hyodong Kim ${ }^{1}$, Sanghun $\mathrm{Lee}^{2}$, Daegu Son ${ }^{3}$, Hyeonjung $\mathrm{Yeo}^{2}$ \\ ${ }^{1}$ Pladen Plastic Surgery Clinic, Seoul; ${ }^{2}$ Department of Plastic and Reconstructive Surgery, Daegu Fatima Hospital, Daegu; ${ }^{3}$ Department of \\ Plastic and Reconstructive Surgery, Keimyung University School of Medicine, Daegu, Korea
}

\begin{abstract}
Background Blepharoplasty has both aesthetic and functional benefits in patients with pseudoptosis; however, previous studies could not demonstrate its beneficial effects quantitatively and objectively. The authors objectively analyzed the visual field before and after surgery and investigated whether measurements of the visual field can be applied as a suitable predictor of surgical outcomes.

Methods In total, 18 eyelids in nine patients with pseudoptosis who had undergone simple skin excision blepharoplasty were evaluated prospectively from February to May 2016. The visual fields were analyzed preoperatively and 3 months postoperatively using the Goldmann kinetic perimetry test. The visual field test area was assessed using Adobe Photoshop.

Results Blepharoplasty had an average 4.99-fold beneficial effect on the superior visual field. In particular, more improvement was seen in the superior temporal quadrant than in the nasal quadrant. No correlation was found between the preoperative margin-to-reflex distance 1 (MRD1) and the surgical outcome $(P=0.119)$. However, there was a strong correlation between the preoperative superior visual field and the surgical outcome $(P=0.001)$.

Conclusions Using the Goldmann kinetic perimetry test, we objectively and quantitatively proved the beneficial effect of blepharoplasty on patients with pseudoptosis. Furthermore, we demonstrated that the preoperative visual field is a better preoperative surgical outcome predictive factor than the preoperative MRD1.
\end{abstract}

Keywords Blepharoplasty / Blepharoptosis / Visual field / Visual disorders

\author{
Correspondences: Daegu Son \\ Department of Plastic and \\ Reconstructive Surgery, Keimyung \\ University School of Medicine, 1035 \\ Dalgubeol-daero, Dalseo-gu, Daegu \\ 42601, Korea \\ Tel: +82-53-258-7817 \\ Fax: +82-53-258-4590 \\ E-mail: handson@dsmc.or.kr \\ Hyeonjung Yeo \\ Department of Plastic and \\ Reconstructive Surgery, Daegu Fatima \\ Hospital, 99 Ayang-ro, Dong-gu, \\ Daegu 41199, Korea \\ Tel: $+82-53-940-7340$ \\ Fax: +82-53-940-7344 \\ E-mail: pleasure-hj@hanmail.net
}

Received: June 17, 2021 - Revised: October 15, 2021 • Accepted: November 6, 2021

pISSN: 2234-6163 • elSSN: 2234-6171 • https://doi.org/10.5999/aps.2021.01109 • Arch Plast Surg 2022;49:19-24

\section{INTRODUCTION}

Pseudoptosis refers to a condition in which the eyelids are normal in position and function; however, the sagging skin on the upper eyelid blocks the visual field, with an appearance similar to ptosis. The degree of pseudoptosis may become worse as the skin sags with age. This also causes brow ptosis, leading to a considerable restriction of the visual field, which is a functional problem [1-4]. Moreover, from a cosmetic perspective, the ar- eas around the eyes are prominent; therefore, signs of aging are easily seen and may prompt individuals to visit a plastic surgery clinic. Hence, it is well known that upper blepharoplasty, which removes the sagging skin, leads to both cosmetic and functional improvement $[5,6]$.

However, most studies have measured functional improvement after upper blepharoplasty in terms of patients' subjective self-reports or the superior margin-to-reflex distance 1 (MRD1) before and after surgery, as an objective measurement [5-8]. Be- 
cause the MRD1 is easy to measure and has clear measurement points, it is a useful indicator for predicting the effects of surgery, but it does not exactly reflect the degree of functional impairment caused by pseudoptosis $[3,9]$.

It seems that after upper blepharoplasty, most patients are more concerned with the cosmetic effects of surgery than with improvement in their visual field. Nevertheless, they often use such expressions as "it's easier to open my eyes," "everything I see looks brighter," and "my vision seems to have become clearer."

In this study, we measured the visual field before and after surgery and objectively evaluated the functional improvement due to upper blepharoplasty through a direct comparison of areas. Representative visual field tests commonly used include Humphrey static perimetry and Goldmann kinetic perimetry. Of these two, patients usually prefer the Goldman kinetic perimetry test, which is known for its efficiency. Hence, we used the Goldman kinetic perimetry test to compare visual field areas before and after surgery [9].

In this study, the Goldman kinetic perimetry test was conducted before surgery and 3 months after surgery in patients with pseudoptosis, and we quantitatively measured the visual field. This study aimed to establish the degree of improvement in the visual field objectively and to determine whether a preoperative visual field examination could predict the degree of functional improvement after upper blepharoplasty.

\section{METHODS}

After obtaining approval from the Keimyung University Dongsan Hospital Institutional Review Board (2015-09-048), we included patients with pseudoptosis who had undergone upper blepharoplasty at Keimyung University Dongsan Medical Center from February 2016 to May 2016, who signed a consent form to participate in the study, and who were eligible for the Goldmann kinetic perimetry test as subjects. Among these subjects, patients with a history of disease or surgery that may interfere with upper eyelid function in addition to pseudoptosis, patients with other ophthalmic diseases, and patients who failed to complete the visual field examination 3 months after surgery were excluded. Finally, nine patients (18 eyes) were selected for this study.

\section{Surgical techniques}

The same surgeon (DS) performed all surgical procedures using a simple skin excision under local anesthesia. We determined the amount of skin excision while blocking any contribution to upper eyelid elevation by the brow to minimize the activation of the frontalis muscle. The amount of skin on the sagging upper eyelid in the sitting position was checked and inci- sion lines were designed. The lower incision line was marked 1 $\mathrm{mm}$ above the double eyelid line, whereas in patients with no double eyelids, it was marked on the transition site between the thinnest and thickest skin. Actual measurements showed that this area was 7-8 $\mathrm{mm}$ above the eyelid margin. Starting from the lacrimal punctum, the medial lower incision line was designed to be marked almost parallel to the eyelid margin, and when it reached the lateral palpebral commissure, it was designed about $20^{\circ}-30^{\circ}$ upward to the lateral orbital rim. The upper incision line was designed with an obtuse angle of $50^{\circ}-70^{\circ}$ from the medial lower incision line. The width of the excision was measured by grasping the sagging skin with forceps, and the angle of the vertex from the lateral side was designed to be about $30^{\circ}-40^{\circ}$. During the design process, the patient was asked to blink to estimate whether the amount of excision was appropriate. The extent of excision should be wider on the lateral side than on the medial side.

The patient was placed on the operating table in a supine position, and the entire face was sterilized and covered with a disinfectant cloth. Next, 1\% lidocaine containing 1:100,000 epinephrine was injected into the incision line. Care was taken to inject the lidocaine and epinephrine mixture just beneath the skin, so that the blood vessels on the surface were not damaged. After the injection, the skin was left for about 7 minutes to ensure hemostasis. Next, an incision was made with a no. 15 surgical knife, only the skin was excised with sharp Metzenbaum scissors, and the orbicularis oculi muscle was preserved. After hemostasis of the surgical site was achieved using electrocautery, there was no further bleeding. Subcutaneous sutures were performed in one place with 5-0 or 6-0 polydioxanone depending on the thickness of the skin between the lateral palpebral commissure and the lateral orbital margin. Next, the skin was sutured in a simple interrupted fashion using 6-0 nylon.

\section{Collection of medical records and analysis}

The following data were collected using medical records and clinical pictures: the patient's age at the time of surgery, medical history and surgical history, the MRD1 before surgery, Goldma$\mathrm{nn}$ kinetic perimetry test records before and after surgery, and the presence of complications at follow-up.

To check the patient's visual field, the visual field function test using Goldmann kinetic perimetry test (Haag 940; Mason, OH, USA) was performed before surgery and 3 months after surgery (Fig. 1). The test was performed with one eye closed for each eye. When the white target of III-4e (size: $4 \mathrm{~mm}^{2}$, intensity: 10 $\mathrm{dB})$ moved from the periphery to the center at about $4^{\circ} / \mathrm{sec}$, the range in which the target was visible on the $15^{\circ}$-wide meridian was recorded, resulting in the Goldmann visual field (GVF) test 

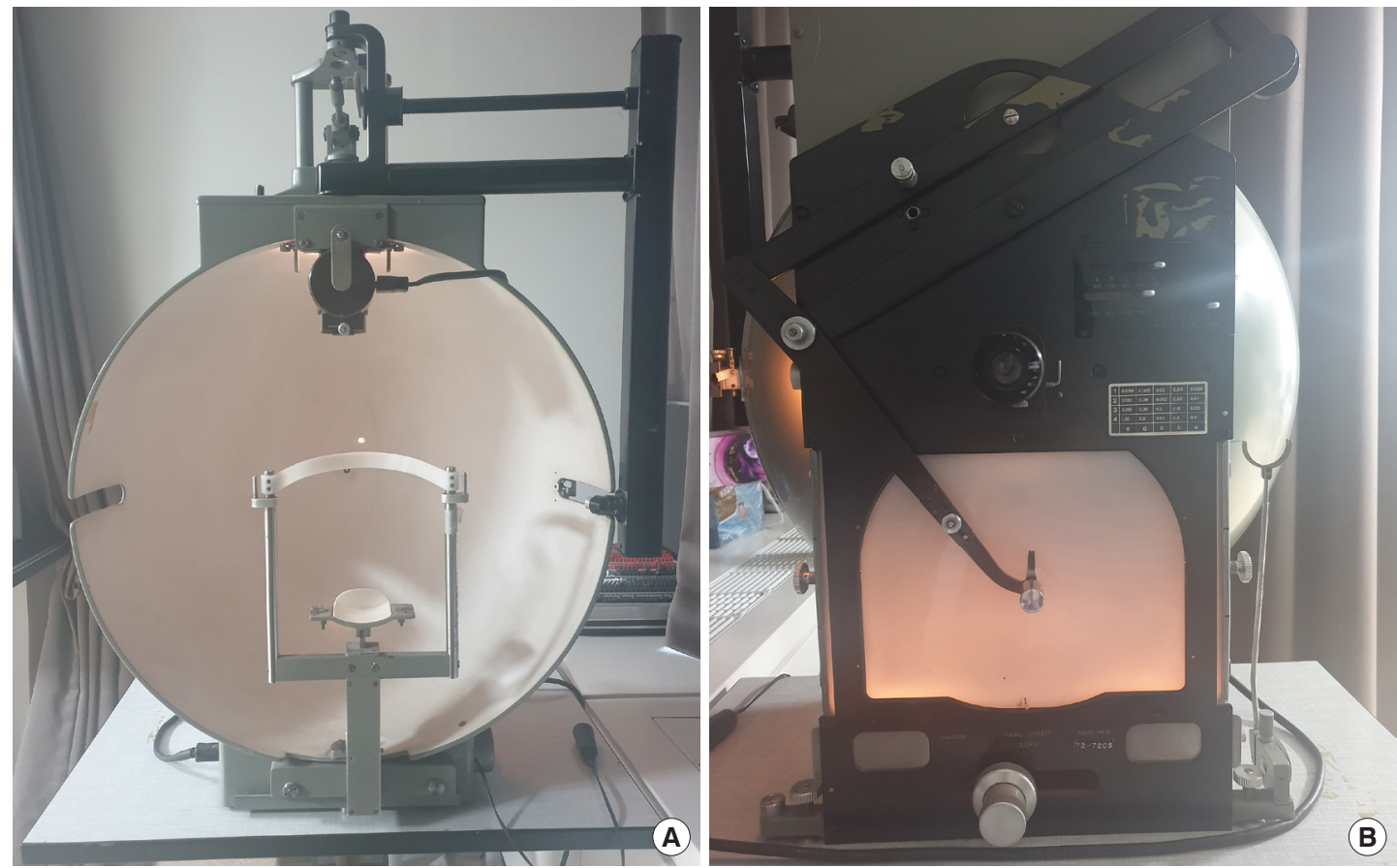

Fig. 1. Goldman perimetry system. (A) Participants' side. (B) Examiner's side.

result sheet (Figs. 2, 3).

All measurements were made using Adobe Photoshop CS6 (Adobe Systems Incorporated, San Jose, CA, USA). First, the GVF result sheet to be measured was scanned and saved as a file with the TIFF extension. Later, the image file was loaded into Photoshop CS6 and the ruler tool was used to determine the distance in pixels from the center point of the GVF to $10^{\circ}$ in any direction. The procedure for this calculation is as follows: go to Image $>$ Analysis $>$ Set Measurement Scale $>$ Custom, enter the pixel value measured above in Pixel Length, set Logical Length to 12, Logical Units to mm, click Save Preset, and save it as "GVF Quantification." Next, using the Magic Wand tool and the Adding and Subtracting functions, select as precisely as possible the area with the $0^{\circ}$ meridian, $90^{\circ}$ meridian, and III-4e isopter as the borders. After completing the zone selection and confirming that Image $>$ Analysis $>$ Set Measurement Scale $>$ GVF Quantification is selected, run Image $>$ Analysis $>$ Record Measurements. The Measurement Log window appears at the bottom of the screen, and the area of the selected area is measured as square millimeters [10].

These values were used as the visual field area for the superior temporal quadrant (STQ) for the right eye and the superior nasal quadrant (SNQ) for the left eye. Similarly, the areas of the parts bordered by the $180^{\circ}$ meridian, $90^{\circ}$ meridian, and III-4e isopter were measured and used as the visual field area for the SNQ for the right eye and the STQ for the left eye.
Using the above method, the SNQ and STQ areas in both eyes were calculated before surgery and 3 months after surgery in all patients. The superior visual field (SVF) of the patient's upper visual field was calculated as $\mathrm{SNQ}+\mathrm{STQ}$.

\section{Statistical analysis}

The preoperative SVF and postoperative SVF were compared using the paired t-test to check whether surgery improved the visual field. In order to quantify the surgical effect, the postoperative improvement in the visual field (postoperative SVF minus preoperative SVF) was divided by the preoperative SVF. Averages and standard deviations were calculated to determine the magnitude of visual field improvement by upper blepharoplasty. The respective relationships of the preoperative MRD1 and preoperative SVF with the surgical effect were compared using linear regression to determine whether these parameters had significant relationships with the outcomes of surgery.

All data were analyzed using SPSS for Windows 22.0 (IBM Corp., Armonk, NY, USA). The results were considered statistically significant at $\mathrm{P}<0.05$.

\section{RESULTS}

We included a total of nine patients (18 eyes) in this study, of whom four (44.4\%) were men and five (55.5\%) were men. The average age of the patients was 59.66 years, and the average 


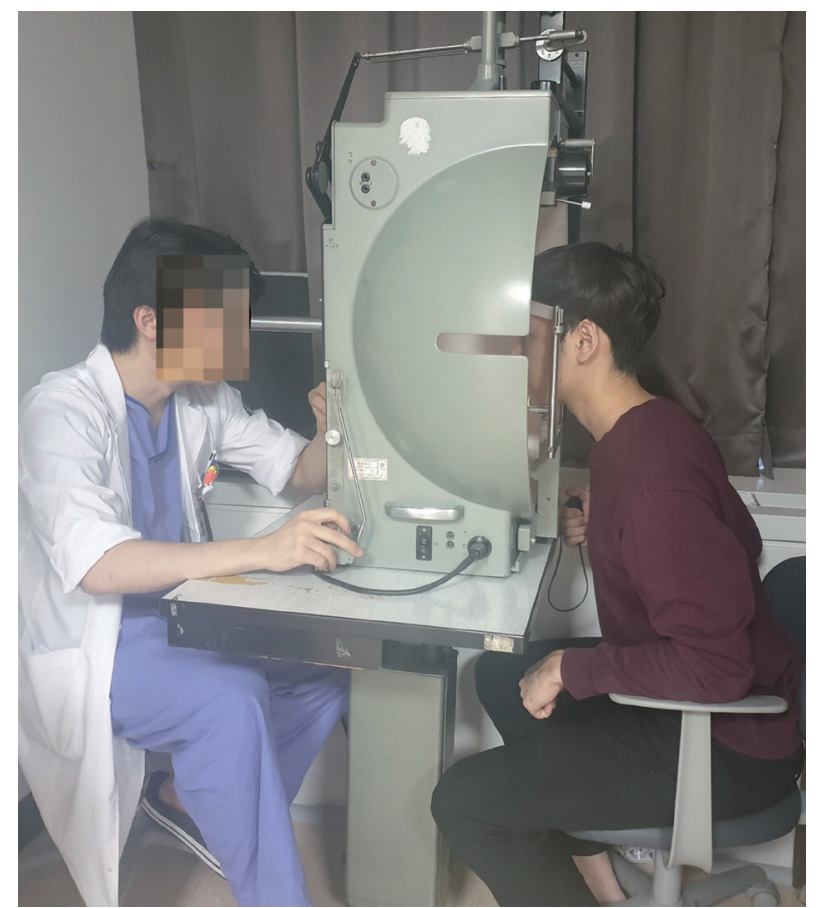

Fig. 2. Goldmann kinetic perimetry test. The image shows a participant seated on the right, facing the dome, and an examiner seated on the left. The examiner controls the position of the target light by moving the projector via a bar with his or her left hand. The bar has a marker on the examiner's side (not shown) that indicates the target light position on a chart in polar coordinates. The target light is presented by pressing a lever with the right hand.

MRD1 before surgery was $1.41 \mathrm{~mm}$. The preoperative mean SNQ, STQ and SVF were $799.55 \mathrm{~mm}^{2}, 906.77 \mathrm{~mm}^{2}$, and $1,706.33 \mathrm{~mm}^{2}$, respectively. The postoperative mean SNQ STQ and SVF were $2,236.66 \mathrm{~mm}^{2}, 2,737.00 \mathrm{~mm}^{2}$, and $4,973.66$ $\mathrm{mm}^{2}$, respectively. The average SNQ STQ and SVF were $1,437.11 \mathrm{~mm}^{2}, 1,830.22 \mathrm{~mm}^{2}$, and $3,267.33 \mathrm{~mm}^{2}$, respectively.

A statistically significant improvement was found in patients' upper visual field following upper blepharoplasty $(\mathrm{P}<0.001)$. The STQ showed a remarkably larger postoperative increase than the SNQ (Table 1).

After blepharoplasty, the visual field improved by 4.99 times on average. The visual field improved by 6 times in patients with a visual field of less than $705 \mathrm{~mm}^{2}$ before surgery and by 2 times in patients with a visual field less than $1,594 \mathrm{~mm}^{2}$.

Preoperative MRD1 and preoperative SVF were linearly regressed on the effect of improvement in the visual field after surgery, and a significant relationship was only found between preoperative SVF and visual field improvement (Figs. 4, 5).

\section{DISCUSSION}

As expected, upper blepharoplasty increased the upper visual
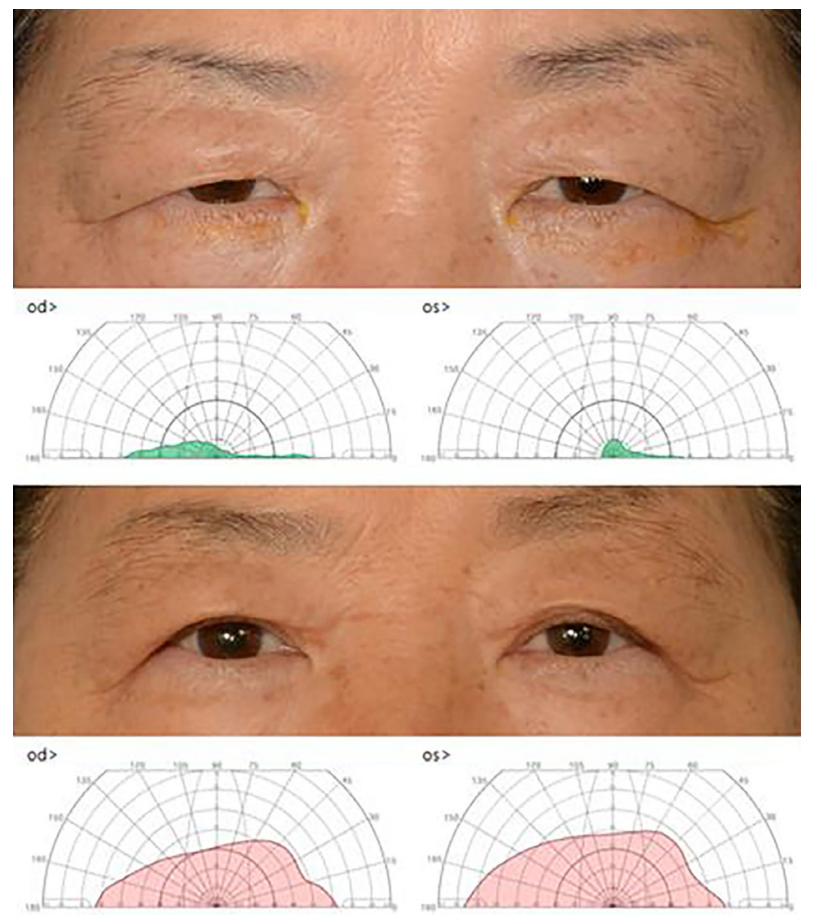

Fig. 3. Preoperative and 3-month postoperative photographs of the patient and preoperative and 3-month postoperative Goldmann kinetic perimetry test results. The area under the green line is the preoperative superior visual field. The area under the red line is the 3-month postoperative superior visual field. OS, oculus sinister (left eye); OD, oculus dexter (right eye).

Table 1. Preoperative and postoperative SVF areas

\begin{tabular}{lccc}
\hline & $\begin{array}{c}\text { Preoperative area } \\
\left(\mathrm{mm}^{2}\right)\end{array}$ & $\begin{array}{c}\text { Postoperative area } \\
\left(\mathrm{mm}^{2}\right)\end{array}$ & P-value \\
\hline SNQ & 799.55 & $2,236.67$ & $<0.001^{\text {a) }}$ \\
STQ & 906.77 & $2,737.00$ & $<0.001^{\text {a) }}$ \\
SVF & $1,706.33$ & $4,973.66$ & $<0.001^{\text {a) }}$ \\
\hline
\end{tabular}

SNQ, superior nasal quadrant; STQ, superior temporal quadrant; SVF, superior visual field.

a) Statistically significant.

field in patients with pseudoptosis. The uniqueness of this study is that it objectively quantified the improvement in the visual field after upper blepharoplasty.

We can summarize facial aging in two words: sagging and deflation. Sagging occurs due to gravity and weakening of the tissue, whereas deflation occurs mainly due to a reduction in collagen and subcutaneous fat. Facial aging appears most prominently around the eyes. Sagging of the eyelids is easily noticeable because the stretched skin covers part of the eyelid margin from the outside. However, aging not only causes sagging of the eyelids, but also ptosis of the eyebrows, which is not easily noticeable. The visual field can be blocked by sagging of the eyebrows and the eyelids, causing the patient to raise his or her eyes using the frontalis muscle to ensure vision. Excessive use of the fronta- 


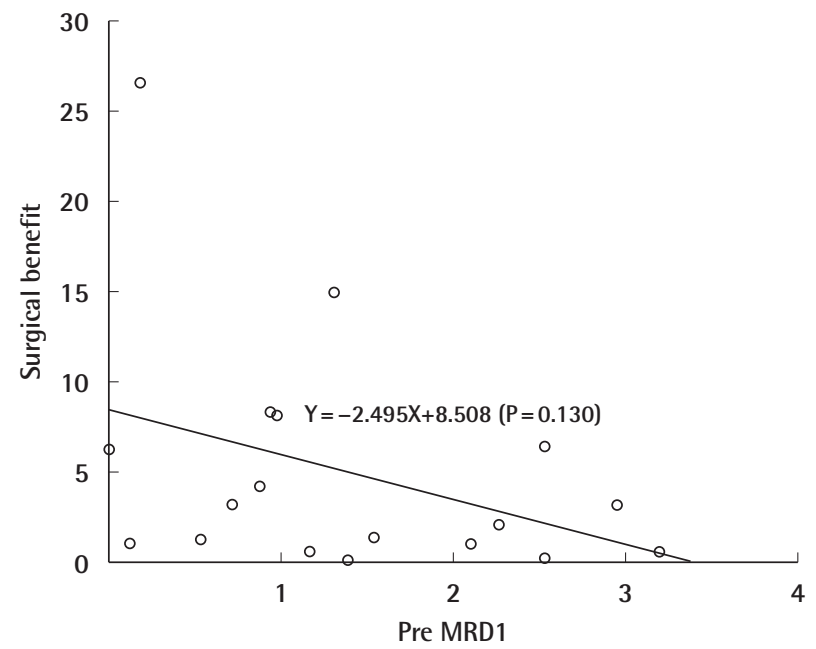

Fig. 4. Scatter diagram showing preoperative margin-to-reflex distance 1 (MRD1) and the functional benefit of surgery (postoperative superior visual field area-preoperative superior visual field area/preoperative superior visual field area).

lis muscles can cause deep wrinkles on the forehead. Hence, patients with ptosis on one upper eyelid tend to develop deep forehead wrinkles only on that side [11].

According to a study by Yun et al. [12], the position of the eyebrows is maintained via a dynamic balance between the frontalis, which raises the eyebrows, and the corrugator supercilii and the orbicularis oculi, which lower the eyebrows; however, aging involves an increase in eyebrow-lowering activity. Therefore, sagging of the eyebrows not only occurs due to weakening of the tissue and gravity, but also results from a dynamic imbalance between the muscles that lower and raise the eyebrows.

There is a view that since eyebrow sagging cannot be corrected by upper blepharoplasty alone, a forehead lift also should be performed simultaneously. According to a study by Kim et al. [13], the eyebrows did not change in position up to 6 months after upper blepharoplasty, but the activity of the frontalis muscles gradually decreased on electromyography. This was probably because the visual field was secured to some extent after upper blepharoplasty and there was no more need for excessive use of the frontalis muscles. Patients' comments after upper blepharoplasty (e.g., "it's easier to open my eyes" and "my vision has become clearer") may reflect the need for less force for the frontalis and the fact that a larger visual field has been secured, as demonstrated in this study. Based on the results from previous research, as well as this study, it seems that cosmetic and functional improvement can be achieved with only upper blepharoplasty without forehead reduction.

The extent of sagging of the eyebrows differs on the internal and external sides. According to a study by Park et al. [14], the medial side does not change significantly even with aging,

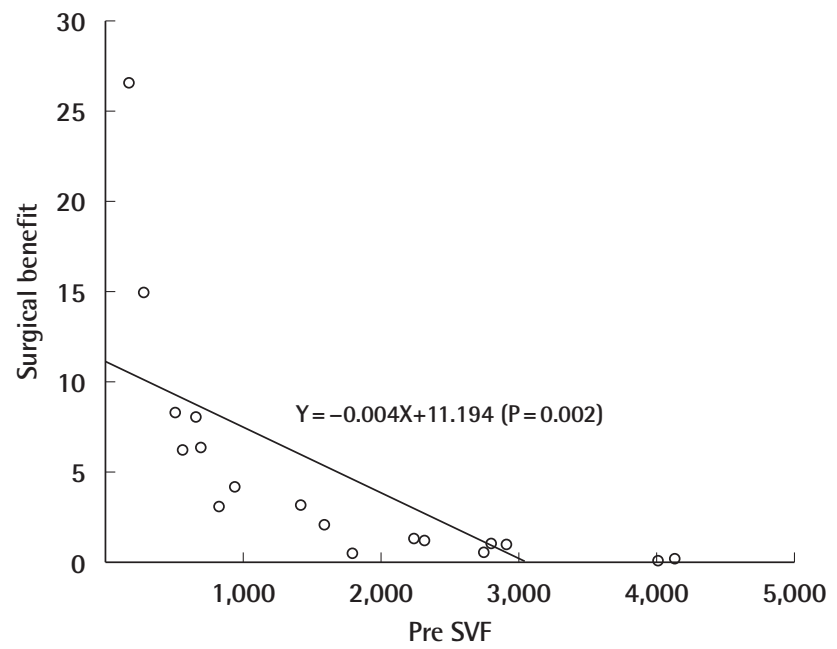

Fig. 5. Scatter diagram showing the preoperative superior visual field (SVF) and the functional benefit of surgery (postoperative superior visual field area-preoperative superior visual field area/preoperative superior visual field area).

whereas it sags extensively on the lateral side. According to the results of this study, the visual field improved more in the STQ than in the SNQ. In this study, the surgical method employed to design the skin excision on the upper eyelid was such that the length of the excision on the lateral side was larger than that on the medial side, and an absorbable subcutaneous suture was added on the lateral side because sagging was more severe on the lateral side.

No significant relationship was found in the present study between the MRD1 before surgery and the amount of visual field improvement, while there was a significant relationship between the SVF before surgery and the amount of visual field improvement. As a preoperative index, the SVF can predict the amount of improvement more accurately than the MRD1. To enable the use of the SVF as a reliable clinical indicator, additional studies are needed with more patients.

A limitation of this study is that we tested the correlations between the MRD1, SVF, and the amount of visual field improvement without considering any other possible factors. Therefore, further studies including more control variables, such as the amount of skin excision and the presence of blepharoptosis, are needed to confirm the relationships between the MRD1, SVF, and visual field improvement. However, even with a small number of patients and a prospective design, this study produced meaningful results. In addition, further research is warranted to create a new test protocol in which the upper visual field areas are automatically calculated based on the SVF area of the Goldmann kinetic perimetry test using a computer.

We evaluated the effect of upper blepharoplasty on functional improvement in patients with pseudoptosis using the Goldma- 
$\mathrm{nn}$ kinetic perimetry test. The improvement effects were measured before surgery and 3 months after surgery to achieve a quantitative and objective evaluation of the effects. Upper blepharoplasty was effective in improving the upper visual field in patients with pseudoptosis. The magnitude of improvement was 4.99-fold on average. The preoperative SVF, but not the preoperative MRD1, showed a statistically significant correlation with functional improvement after surgery. In patients with pseudoptosis, measuring the patient's upper visual field using the Goldmann kinetic perimetry test before upper blepharoplasty seems to be useful for predicting and evaluating postoperative results.

\section{NOTES}

\section{Conflict of interest}

No potential conflict of interest relevant to this article was reported.

\section{Ethical approval}

The study was approved by the Institutional Review Board of Keimyung University Dongsan Hospital (IRB No. 2015-09048) and performed in accordance with the principles of the Declaration of Helsinki. Written informed consent was obtained.

\section{Patient consent}

The patients provided written informed consent for the publication and the use of their images.

\section{Author contribution}

Conceptualization: D Son. Data curation: H Kim. Writing original draft: H Kim. Writing - review \& editing: S Lee, D Son, $\mathrm{H}$ Yeo. All authors read and approved the final manuscript.

\section{ORCID}

Hyodong Kim

Sanghun Lee

Daegu Son

Hyeonjung Yeo

\section{REFERENCES}

1. Carraway JH, Tran P. Blepharoplasty with ptosis repair. Aes- thet Surg J 2009;29:54-61.

2. An SH, Jin SW, Kwon YH, et al. Effects of upper lid blepharoplasty on visual quality in patients with lash ptosis and dermatochalasis. Int J Ophthalmol 2016;9:1320-4.

3. Cahill KV, Bradley EA, Meyer DR, et al. Functional indications for upper eyelid ptosis and blepharoplasty surgery: a report by the American Academy of Ophthalmology. Ophthalmology 2011;118:2510-7.

4. Lim JM, Hou JH, Singa RM, et al. Relative incidence of blepharoptosis subtypes in an oculoplastics practice at a tertiary care center. Orbit 2013;32:231-4.

5. Huijing MA, van der Palen J, van der Lei B. The effect of upper eyelid blepharoplasty on eyebrow position. J Plast Reconstr Aesthet Surg 2014;67:1242-7.

6. Kiang L, Deptula P, Mazhar M, et al. Muscle-sparing blepharoplasty: a prospective left-right comparative study. Arch Plast Surg 2014;41:576-83.

7. Hintschich C. Periocular plastic surgery. Dtsch Arztebl Int 2010;107:141-6.

8. Latting MW, Huggins AB, Marx DP, et al. Clinical evaluation of blepharoptosis: distinguishing age-related ptosis from masquerade conditions. Semin Plast Surg 2017;31:516.

9. Ural O, Mocan MC, Dolgun A, et al. The utility of marginreflex distance in determining the type of surgical intervention for congenital blepharoptosis. Indian J Ophthalmol 2016;64:752-5.

10. Zahid S, Peeler C, Khan N, et al. Digital quantification of Goldmann visual fields (GVFs) as a means for genotypephenotype comparisons and detection of progression in retinal degenerations. Adv Exp Med Biol 2014;801:131-7.

11. Zoumalan CI, Lisman RD. Evaluation and management of unilateral ptosis and avoiding contralateral ptosis. Aesthet Surg J 2010;30:320-8.

12. Yun S, Son D, Yeo H, et al. Changes of eyebrow muscle activity with aging: functional analysis revealed by electromyography. Plast Reconstr Surg 2014;133:455e-463e.

13. Kim D, Son D, Kim M, et al. Does upper blepharoplasty affect frontalis tonicity? J Plast Reconstr Aesthet Surg 2015; 68:638-44.

14. Park J, Yun S, Son D. Changes in eyebrow position and movement with aging. Arch Plast Surg 2017;44:65-71. 